\title{
Observation of Counterpart Radical on Main-Chain Scission in Plasma-Irradiated Methacrylic Polymers
}

\author{
Masayuki KuzuYa, ${ }^{*}$ Kazuhiko SaWada, Tomokazu TAKaI \\ and Akihiro NoGUCHI \\ Laboratory of Pharmaceutical Physical Chemistry, Gifu Pharmaceutical University, \\ 5-6-1, Mitahora-higashi, Gifu 502, Japan
}

(Received July 22, 1992)

\begin{abstract}
We describe the extended study of plasma-induced radicals in two methacrylic polymers which were strictly dried at higher temperature and for a longer time than before. It has been shown that the present electron spin resonance (ESR) spectra of plasma-irradiated methacrylic polymers are appreciably different from those observed previously, and are characterized by the presence of many small peaks on the outer side of major peaks. Computer simulations disclosed that the difference is caused by the presence of a fifteen-line component spectrum (V) of a previously unobserved radical (5) which should be produced by the addition of hydrogen radical to the counterpart isobutene-like moiety of the terminating radical (1).
\end{abstract}

KEY WORDS Plasma-Irradiation / Solid Radical / Methacrylic Polymer / Electron Spin Resonance Spectroscopy /

As part of our continuing work on the elucidation of plasma-induced radicals of organic polymers, ${ }^{1-9}$ we recently reported the ESR studies of the radicals in four kinds of plasma-irradiated powdered methacrylic polymers (acrylic resins), poly(methacrylic acid) (PMAA), its methyl ester (PMMA), and two types of their copolymers of MMA and MAA, commercially known as Eudragit L100 $(6: 4)$ and Eudragit S100 (7:3). ${ }^{4}$ All the observed spectra were quite satisfactorily reproduced by the systematic computer simulations invoking four kinds of isotropic component spectra, nine-line spectrum (I), doublet (II), seven-line spectrum (III) and broad single line spectrum (IV). These component spectra were assigned to the terminating radical (1), mid-chain radical (2), monomer-derived radical (3), and immobilized dangling bond sites (4), respectively. The fact that all the component radicals in the powder spectra can be approximated by the isotropic lines is apparently due to the motional narrowing.

It has also been shown that the mechanism by which the terminating radical (1) is formed involves an initial elimination of carboxyl group, followed by depolymerization of the polymer main-chain by $\beta$-scission. In this event, the isobutene-like moiety should also have been simultaneously formed as the counterpart of the initial $\beta$-scission as shown in Scheme 1.

However, we found no evidence for the radical formation derived from a reaction of

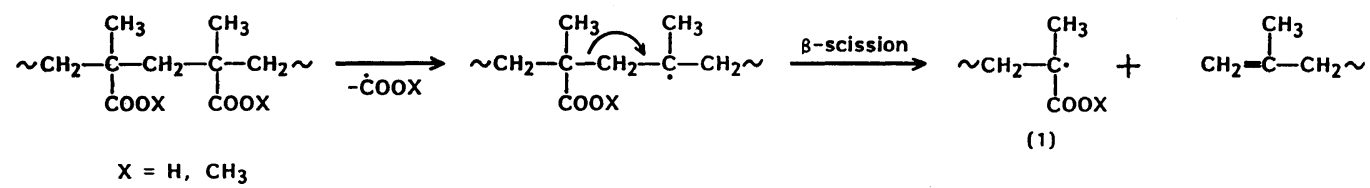

Scheme 1. 
such a moiety with a hydrogen radical, although the regenerated monomer did undergo such hydrogen radical addition reactions to give the seven-line spectrum (III) in the ESR spectrum. This may be accounted for by the high degree of depolymerization, resulting in the regeneration of a large amount of monomers. Consequently, a stoichiometrical amount of the vinyl bond of isobutene-like moiety is uncompetitively lower than that of regenerated monomers.

We found that such radicals, in fact, were formd and detected by the ESR spectrum, when methacrylic polymers used for plasmairradiation were more strictly dried than before.

\section{EXPERIMENTAL}

\section{Materials}

Polymer samples were commercially available, and purified by dissolving them in good solvents (in chloroform for PMMA, and in methanol for PMAA and Eudragit L100), precipitating in excess methanol, ether, and water, respectively, and then filtering. This procedure was repeated twice. After drying in vacuo at $70^{\circ} \mathrm{C}$ for $10 \mathrm{~h}$, the polymers were pulverized and screened with a 200 mesh sieve. The mean particle size of each powdered sample was measured in tetrahydrofuran by Coulter Counter (Model TA-2, Coulter Electronics Inc. U.S.A.), from which the specific surface area $\left(\mu \mathrm{m} 2 \times 10^{4}\right)$ was determined: 6.05 for PMMA and 6.38 for Eudragit L100. All the spectroscopic data (DSC, IR, and NMR) of the present samples were identicl with those previously used $\left(60^{\circ} \mathrm{C}\right.$ for $5 \mathrm{~h}$ in vacuo $) .^{4}$ The molecular weights of the powdered samples thus obtained were determined by GPC (Shimadzu LC-6A), equipped with gel column (Shodex pre-column KD-800P (4.6 mm diameter $\times 1 \mathrm{~cm})$ and Shodex KD-80M $(8.0 \mathrm{~mm}$ diameter $\times 30 \mathrm{~cm})$ and a refractive index detector (Shimadzu, RID-6A) under the following conditions: column temperature, $40^{\circ} \mathrm{C}$; elution

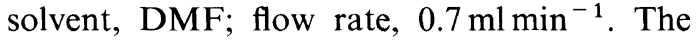
calibration for the molecular weight determination was made by standard specimen of polyethylene oxide; $\bar{M}_{n} \doteqdot 245000, \bar{M}_{w} \doteqdot 335000$ $\left(\bar{M}_{w} / \bar{M}_{n}\right.$ Eudragit L100).

\section{ESR Spectral Measurements}

Powdered samples $(50 \mathrm{mg})$ were placed in a specially-designed ampule $(30 \mathrm{~mm}$ i.d., $100 \mathrm{~mm}$ long) connected to a capillary tube ( $2 \mathrm{~mm}$ i.d.) at the uppermost part of the ampule. The ampule was filled with argon gas for plasmolysis ( 0.5 Torr) and sealed. The plasma state was sustained for the prescribed period of time with stirring of samples at room temperature by a radio frequency discharge of inductive coupling at $13.56 \mathrm{MHz}$ with the supplied power $(40 \mathrm{~W})$, and the ESR measurements were performed while turning the ampule upside down after plasma-irradiation, which is fundamentally the same procedure as that reported earlier. ${ }^{4}$

The ESR spectral intensity was determined by double integration. Measurements of $g$ values were made relative to the fourth signal from the lower magnetic field $(g=1.981)$ of $\mathrm{Mn}^{2+}$ in $\mathrm{MgO}$. The radical concentration (spin numbers $/ \mathrm{cm}^{2}$ ) was calculated with the aid of calibration lines obtained from PMMA powdered sample impregnated with DPPH. ESR spectra were recorded by a JES-REIX (JEOL) spectrometer with X-band and 100 $\mathrm{kHz}$ field modulation, and the microwave power level was kept at less than $0.04 \mathrm{~mW}$, since the power levels higher than this began to produce saturation effects in the spectra.

\section{Computer Simulations}

Computer simulations were performed on a 32-bit microcomputer (NEC PC-9801RA) by fitting iteratively the spectroscopic parameters ( $g$-value, line width at half-height (HV), hyperfine splitting constant (HSC), and relative spectral intensity of each component radical) with the observed spectra (digitized) according to non-linear least squares meth- 
od. ${ }^{4}$ The trial spectroscopic parameters of radical (5) were taken from that of polyisobutene radical. ${ }^{12,13}$

\section{RESULTS AND DISCUSSION}

\section{Observed ESR Spectra}

Progressive changes in room temperature ESR spectra of argon plasma-irradiated PMMA and Eudragit L100 as a function of plasma duration are shown in Figure 1.

It is seen that the present spectral features are apparently different in detailed points from those observed previously, which are shown in Figures 2 for a comparison. Both spectra in Figure 1 appear to be of larger small peaks on the outer side of both higher- and lower-field of main peaks, and show large de- viation from the standard isotropic nine-lines spectrum.

\section{Simulated Spectra}

Figure 3 shows the simulated ESR spectra corresponding to Figure 1. It can be seen that all the observed spectral features including small outermost peaks have been nicely reproduced by these simulations. The simulated spectra consist principally of five kinds of isotropic spectra; nine-line spectrum (I), doublet (II), seven-line sepectrum (III), broad singlet-like spectrum (IV), and fifteen-line spectrum (V). The component spectra, (I)(IV), are all essentially identical with those obtained previously, ${ }^{4}$ but the spectrum $(\mathrm{V})$ is a new component spectrum unique to the present samples. A representative spectral fea-
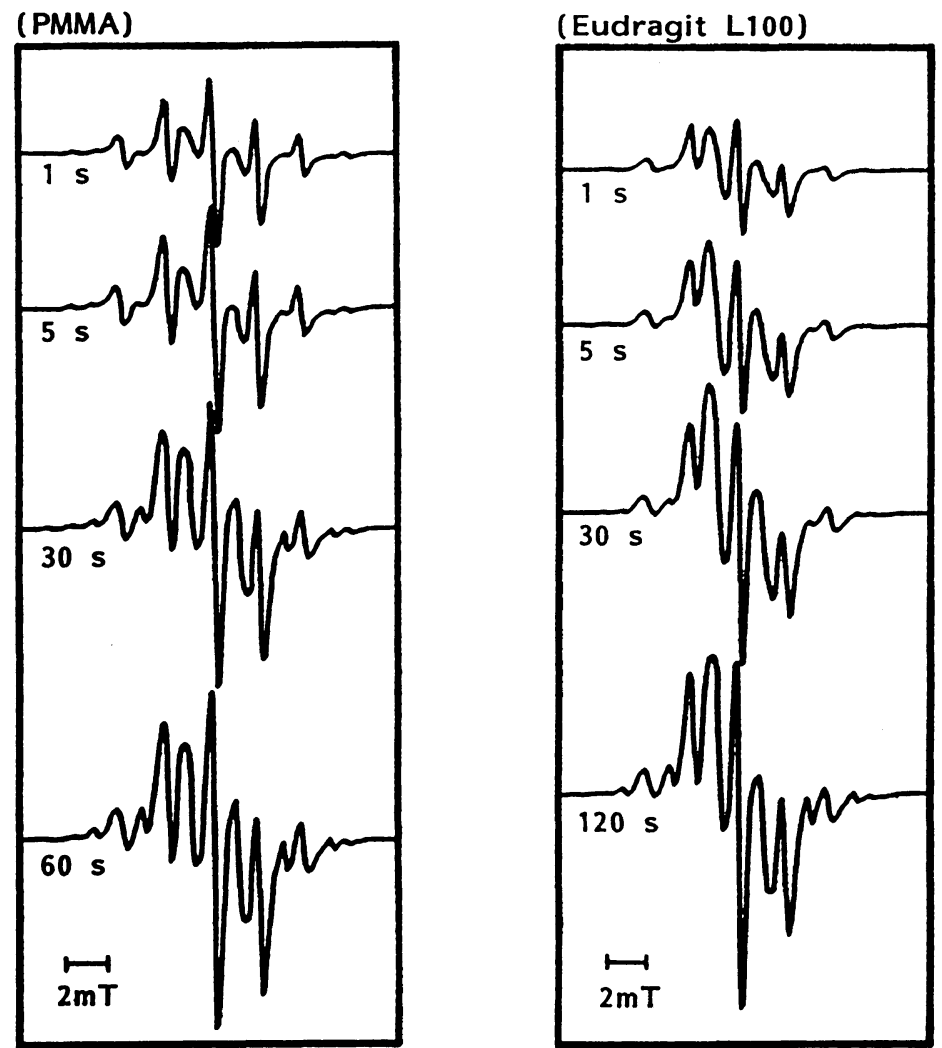

Figure 1. Observed ESR spectra of Ar plasma-irradiated powders of strictly-dried PMMA and Eudragit L100 with various plasma duration. 

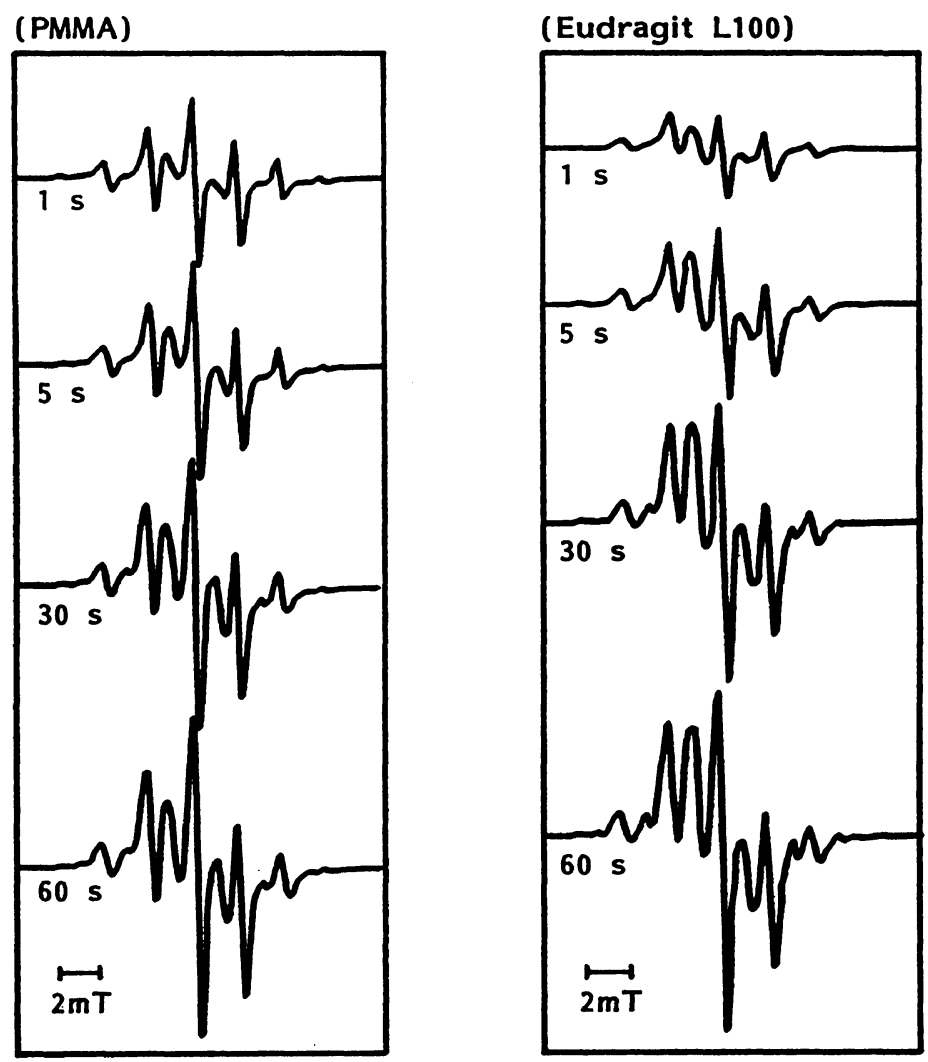

Figure 2. Observed ESR spectra of Ar plasma-irradiated powders of PMMA and Eudragit L100 with various plasma duration observed previously. ${ }^{4}$

ture for the component spectrum (V) in the simulated spectra is shown in Figure 4.

The ESR spectroscopic data of the radical (V) are as follows: $g=2.0038, a_{\beta}^{\mathrm{Me}}=2.23 \mathrm{mT}$ $(6 \mathrm{H}), a_{\beta}^{\mathrm{CH}_{2}}=1.2 \mathrm{mT}(2 \mathrm{H})$ for PMMA, $g=$ $2.0033, a_{\beta}^{\mathrm{Me}}=2.23 \mathrm{mT}(6 \mathrm{H}), a_{\beta}^{\mathrm{CH}_{2}}=1.2 \mathrm{mT}(2 \mathrm{H})$ for Eudragit L100. Thus, further deviation of the present observed spectra from the standard nine-line spectrum was confirmed due to the presence of fifteen-line spectrum (V) of an additional component radical. We emphasize that all the satifsactory simulated spectra including small outermost peaks were consistently obtained only by invoking all the abovementioned component spectra with differing the ratio (vide infra).

\section{ESR Kinetics}

Figure 5 shows the ESR kinetics of component radical formation as a function of plasma duration based on the results of simulations.

It is seen that all component radicals exhibit parabolic increases in intensity on both polymers, and the copolymer Eudragit L100 is much more plasma sensitive than the homopolymer PMMA in the radical formation as in the case of previous samples. ${ }^{4}$ However, the present result differs not only in the presence of fifteen-line spectrum but also decrease in intensity of nine-line spectrum (I) and seven-line spectrum (III) for both methacrylic polymers (1.2/2.3 of (I) and $0.38 / 0.47$ of (III) in PMMA, $6.4 / 7.5$ of (I) and $0.54 / 1.25$ of (III) in Eudragit L100). 

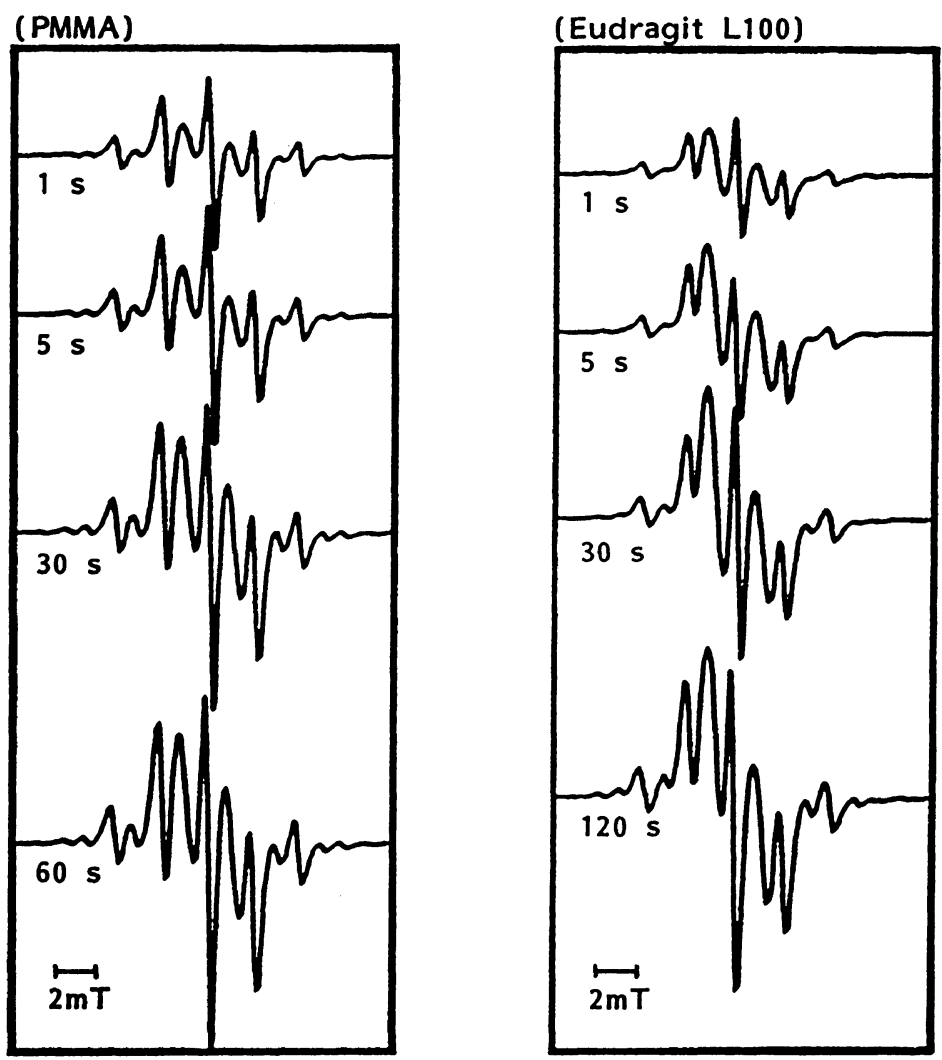

Figure 3. Simulated ESR spectra corresponding to observed spectra in Figure 1.

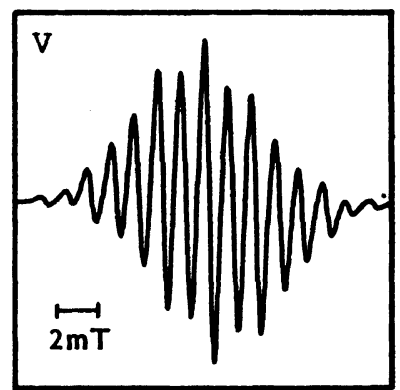

Figure 4. Representative spectral features for fifteen-line component spectrum in simulated spectra.

\section{Radical Structure}

Since the component spectra, (I)-(IV), are all essentially identical with those observed previously, they can be assigned to the terminating radical (1), mid-chain radical (2), monomer-derived radical (3), and dangling bond sites (4), respectively.

It is known that the terminating radical, $-\mathrm{CH}_{2}-\dot{\mathrm{C}}\left(\mathrm{CH}_{3}\right)_{2}$, is produced in $\gamma$-irradiated polyisobutene, whose ESR spectrum exhibits the fifteen-line spectrum of non-binominal intensity distribution with unequal spacings. ${ }^{12,13}$ This spectrum stems from the fortuitous superposition of six lines causing the spectral line reduction of twenty one-line to fifteen-line spectrum.

As stated in the Introduction, the pathway to generate the terminating radical of methacrylic polymers by $\beta$-scission also produces the terminating isobutene-like moiety having double bonds (Scheme 1), and the addition of hydrogen radicals to this double bond is likely to occur to produce a radical such as $-\mathrm{CH}_{2}-\mathrm{C}\left(\mathrm{CH}_{3}\right)_{2}$. It can be reasonably considered that the spectrum of this type radical 

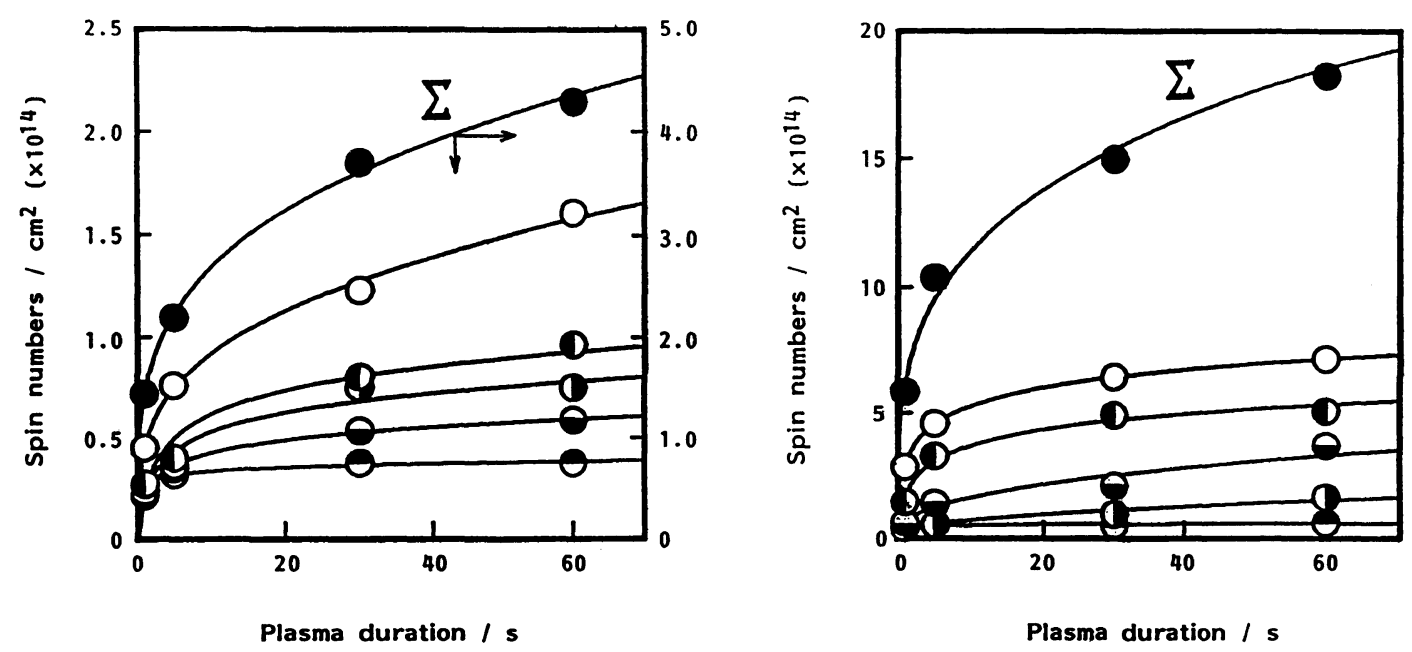

Figure 5. Simulated progressive changes in each of component spectra of Ar plasma-irradiated powders of PMMA (A) and Eudragit L100 (B). O, (I); D, (II); Ө, (III); ๑, (IV); O, (V).

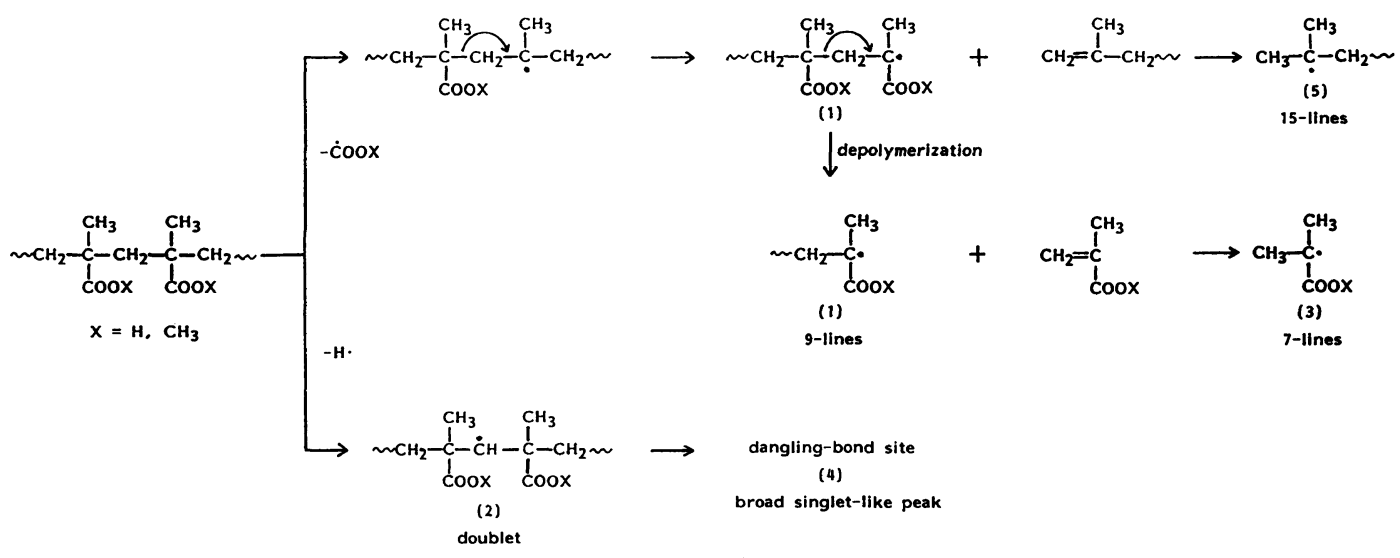

Scheme 2.

should be very similar or essentially identical with that of the terminating radical of polyisobutene. Thus, the present fifteen-line ESR spectra (V) were assigned to the terminating radicals (5) with the addition of hydrogen radicals to the double bonds of the isobutene-like moiety. The spectroscopic parameters deduced from the simulated spectra are also compatible with those shown previously. ${ }^{12,13}$ The free radical electron on the radical (5) should be capable of coupling with two $\beta$-hydrogens as well as one $\alpha$-hydrogen. The isotropic hyperfine constant for $\beta$-hydro- gens depends on the radical conformation, i.e., the rotation angle along the $\mathrm{C}_{\alpha}-\mathrm{C}_{\beta}$ bond.

It is well known $\beta$-hyperfine constant of alkyl radicals can be effectively described by the cosine-square rule, which can be expressed as $a_{\beta}^{\mathrm{H}}=A q \cos ^{2} \theta$, where $\theta$ is the angle between the $\mathrm{H}-\mathrm{C}_{\beta}-\mathrm{C}_{\alpha}$ plane and the axis of $p$-orbital of the free electron, $\mathrm{A}$ is a proportional constant and $q$ is a spin density of the radical center. Accordingly, the rather small and identical values of $\beta$-hydrogen hyperfine constants for methylene groups in the radical (5) are apparently caused by the conformation 
with $\theta \doteqdot 60$ for two $\beta$-hydrogens.

Structural assignments and the mechanism by which all the radicals are formed are summarized in Scheme 2.

\section{CONCLUSION}

The present results demonstrated that the drying condition of the methacrylic polymer samples affect the shape of ESR spectra observed due mainly to the formation of counterpart terminating radical, $-\mathrm{CH}_{2}-\mathrm{C}\left(\mathrm{CH}_{3}\right)_{2}$, (5).

Plasma-irradiation has considerably local thermal effect on polymer surface. This effect may be enhanced on the present polymers thought to be less water-contained or waterfree. The monomers regenerated, therefore, may be effectively leached out of the polymer surface resulting in a significant reduction in monomer quantity, so that the fifteen-line spectrum (V) becomes observable in the present spectrum. This view is consistent with the decrease in intensity of the observed seven-line spectrum (III) of monomer-derived radical (3) in the present polymers.

Because of the intrinsic properties of acrylic resins to form readily the inclusion complex with its monomer and some other solvent, the nature of plasma-induced radical formation in acryrlic resins is reflected in a very sensitive manner by a small content of water included in such polymers. But, to what extent is not yet known.

The present results contribute to our understanding of the nature of the radical for- mation on methacrylic polymers by plasmairradiation, and reinforce the mechanism proposed in a previous paper. ${ }^{4}$

\section{REFERENCES}

1. M. Kuzuya, A. Koide, A. Ito, and A. Noguchi, Chem. Lett., 555 (1989).

2. M. Kuzuya, M. Ishikawa, A. Noguchi, H. Ito, K. Kamiya, and T. Kawaguchi, J. Mater. Chem., 1, 387 (1991).

3. M. Kuzuya, A. Noguchi, H. Ito, S. Kondo, and N. Noda, J. Polym. Sci., Polym. Chem., 29, 1 (1991).

4. M. Kuzuya, A. Noguchi, M. Ishikawa, A. Koide, K. Sawada, A. Ito, and N. Noda, J. Phys. Chem., 95, 2398 (1991).

5. M. Kuzuya, H. Ito, S. Kondo, N. Noda, and A. Noguchi, Macromolecules, 24, 6612 (1991).

6. M. Kuzuya, K. Kamiya, and K. Sawada, Proc. Jpn. Sym. Plasma Chem., 4, 317 (1991).

7. M. Kuzuya, M. Ishikawa, A. Noguchi, K. Sawada, and S. Kondo, J. Polym. Sci., Polym. Chem., 30, 379 (1992).

8. M. Kuzuya, N. Noda, S. Kondo, K. Washino, and A. Noguchi, J. Am. Chem. Soc., 114, 6505 (1992).

9. M. Kuzuya, S. Kondo, H. Ito, and A. Noguchi, Appl. Sur. Sci., 60/61, 416 (1992).

10. M. Kuzuya, K. Kamiya, Y. Yanagihara, and Y. Matsuno, J. Plasma Sources Sci. Technol., in press.

11. For review: (a) M. Kuzuya, "Electron Spin Resonance Study in Solid State Radicals Induced by Plasma Irradiation," in "Trends in Physical Chemistry" Vol. 2, Council of Scicence Research Integration, India, 1991, pp 39-61. (b) M. Kuzuya; Plasma-Photochemistry of Polymers and Its Application for Drug Delivery System, Vol. 5, J. Photopolym. Sci. Technol.," 1992, pp 407-416.

12. E. N. Teleshov, V. A. Sharpatyi, A. N. Pravednikov, and S. S. Medvedev, Zh. Strukt. Khim., 5, 627 (1964).

13. S. Nagai, S. Ohnishi, and I. Nitta, Bull. Chem. Soc. Jpn., 45, 1934 (1972). 